\title{
N-terminal pro B-type natriuretic peptide (NT-proBNP): a possible surrogate of biological age in the elderly people
}

\author{
Antonio Muscari (iD - Giampaolo Bianchi • Paola \\ Forti • Donatella Magalotti • Paolo Pandolfi • Marco \\ Zoli • The Pianoro Study Group
}

Received: 9 April 2020 / Accepted: 21 July 2020 / Published online: 11 August 2020

(C) The Author(s) 2020

\begin{abstract}
NT-proB-type natriuretic peptide (NTproBNP) increases with age and is associated with all-cause mortality. With this study, we assessed its possible utilization as a marker of biological age in comparison with other variables. The study included 1079 non-institutionalized elderly subjects (mean age $72.8 \pm 5.5$ years, 561 women). Baseline measurements were performed of serum NTproBNP and of some laboratory variables previously utilized to estimate biological age
\end{abstract}

\author{
A. Muscari · G. Bianchi · P. Forti · M. Zoli $(\bowtie)$ \\ Department of Medical and Surgical Sciences, University of \\ Bologna-S.Orsola-Malpighi Hospital, Via Albertoni, 15, \\ 40138 Bologna, Italy \\ e-mail: marco.zoli@unibo.it
}

\author{
A. Muscari \\ e-mail: antonio.muscari@unibo.it \\ G. Bianchi \\ e-mail: giampaolo.bianchi@unibo.it \\ P. Forti \\ e-mail: paola.forti@unibo.it \\ D. Magalotti \\ Medical Department of Continuity of Care and Disability, \\ S.Orsola-Malpighi Hospital, Bologna, Italy \\ e-mail: donatella.magalotti@aosp.bo.it \\ P. Pandolfi \\ Epidemiological and Health Promotion Unit, Department of \\ Public Health, AUSL Bologna, Bologna, Italy \\ e-mail: paolo.pandolfi@ ausl.bologna.it
}

(creatinine, albumin, C-reactive protein, cholesterol, blood glucose, leukocytes, lymphocytes, hemoglobin, mean cell volume). During 7 years of follow-up, 114 all-cause deaths occurred. The logarithm of NT-proBNP was the most age-related parameter $(r=0.35, P<0.0001)$. Its relationship with mortality, according to Cox regression and ROC curve (AUC $=0.707$, 95\% CI 0.654-0.759), was stronger than that of all other variables, including age. In multivariate analysis, only NTproBNP and age remained independently associated with mortality. The regression lines between age and NT-proBNP $(\mathrm{pg} / \mathrm{ml})$ allowed a separate estimation of biological age ("proBNPage") for men $(=[\log (\mathrm{NT}-$ proBNP $)+1.2068] / 0.0827)$ and for women $(=[\log (\mathrm{NT}-$ proBNP$)-1.5258] / 0.0478)$. The hazard ratio of all-cause mortality for the fifth quintile of proBNP age ( $\geq 85$ years) compared with the first quintile (<61 years) was $7.9(95 \%$ CI 3.6-17.5). Similarly, the difference between pro-BNPage and chronological age was associated with a hazard ratio of 3.5 in the fifth quintile (95\% CI 1.9-6.4) and was associated with disease count $(P$ for trend $=0.0002)$. In conclusion, NTproBNP was the best indicator of biological age, which can be estimated by simple formulas and might be used for prognostic purposes or as a surrogate end point in epidemiological and intervention studies.

Keywords NT-proBNP · Mortality · Biological age $\cdot$ Epidemiological studies 


\section{Introduction}

The difference between biological and chronological age is easily understandable to everybody, as we all know persons who show more or fewer years than their age and who behave accordingly. Biological age may be defined as a measure, more accurate than chronological age, of how really close we are to death. Finding an enough precise method to determine biological age is more difficult. However, this finding could lead to two important applications: (1) a reliable prognostic index and (2) a surrogate end-point to be used in the search for anti-aging treatments in the place of mortality, which can only be determined with long longitudinal studies.

Keeping in mind these premises, a few characteristics have been defined for the ideal indicator of biological age (Jylhävä et al. 2017; Johnson 2006): (1) it must be correlated with chronological age; (2) it must be associated with mortality, possibly better than chronological age; (3) it must be associated with morbidity; (4) it should be associated with the aging process, rather than with the effects of diseases; (5) it should be easily and repeatedly measurable; (6) it should have a chance to change due to therapeutic interventions.

The studies on this subject have been numerous, suggesting several potential indicators of biological age. These may be grouped into 6 main categories:

1. Measures of DNA methylation (epigenetic clock) (Hannum et al. 2013; Horvath 2013), the indicators most correlated with chronological age, probably because DNA methylation is a time-dependent process. They have however a mild-to-moderate association with mortality, with better results with cancer than with cardiovascular mortality (Chen et al. 2016; Perna et al. 2016)

2. Telomere length in leukocytes (Sanders and Newman 2013), perhaps the most popular and reliable indicator of biological age, although not routinely measurable

3. Proteomic and metabolomic indices (Menni et al. 2015; Johnson et al. 2019), which however are supported by still scarce and poorly conclusive studies

4. Magnetic resonance imaging to assess brain age, which is associated with cognitive decline and biological age (Cole et al. 2018; Elliott et al. 2019)

5. Composite frailty or deficit indices based on the combination of many clinical-anamnestic- functional variables (Mitnitski et al. 2001; Abete et al. 2017; Rockwood et al. 2017; Kim and Jazwinski 2015)

6. Composite indices mainly based on the combination of laboratory variables (Yoo et al. 2017; Belsky et al. 2017; Liu et al. 2018; Waziry et al. 2019). These are easily and repeatedly measurable, including routine variables such as albumin, creatinine, blood glucose, uric acid, C-reactive protein, and leukocytes, many of which, even when considered alone, are associated with mortality, morbidity, and chronological age.

As far as the latter category is concerned, rather surprisingly, some very promising biochemical parameters have not been considered yet. These are natriuretic peptides, particularly B-type natriuretic peptide (BNP) and the N-terminal fragment of its precursor (NTproBNP). Both are mainly released by stressed cardiomyocytes in cardiac walls and are therefore important indicators of heart disease and cardiovascular mortality (McDonagh et al. 2004; Galvani et al. 2004; Ponikowski et al. 2016; Wang et al. 2004). However, they also predict mortality in subjects free of heart disease (McKie et al. 200; Wannamethee et al. 2011; Muscari et al. 2013), are associated with noncardiovascular and all-cause mortality (Muscari et al. 203) and progressively increase with age (Redfield et al. 2002; Wang et al. 2002; Luchner et al. 2013). Within the Pianoro Study, an epidemiological investigation concerning the elderly population resident in three rural municipalities of Northern Italy, we have previously reported the association between baseline NT-proBN $P$ values and mortality during a 7-year follow-up (Muscari et al. 2013). In the present study, we have assessed how NT-proBNP and some biochemical variables utilized in previous studies were associated with chronological age and all-cause mortality, with the goal of identifying, among them, the best indicator of biological age in the elderly population.

\section{Methods}

Subjects

The Pianoro Study has been described in previous publications (Muscari et al. 2007; 2013). Shortly, starting from November 2003, all of the 3255 inhabitants of the 
Pianoro municipality (Northern Italy) aged $\geq 65$ years were invited to participate in the study. Two thousand twenty-two subjects returned a preliminary postal questionnaire and, of them, 1163 agreed to be subsequently examined in our laboratories. The characteristics of the subjects who returned their postal questionnaires, but did not participate in the second phase, have previously been reported (Muscari et al. 2007). Forty-eight subjects were excluded due to missing data and further 36 subjects with a history of heart failure or atrial fibrillation (the main conditions associated with high NT-proBNP levels) were also excluded. Finally, 1079 subjects aged 65-93 years at the time of enrolment (mean age \pm SD $72.8 \pm 5.5$ years, 518 men and 561 women) remained available for our analyses.

This study was approved by our joint UniversityHospital Ethical Committee, and all participants provided their signed informed consent.

\section{Baseline variables}

The subjects with a history of diabetes and those with fasting blood glucose $\geq 126 \mathrm{mg} / \mathrm{dl}$ were considered diabetic. The subjects under lipid lowering treatment and/ or with serum levels of total cholesterol $\geq 200 \mathrm{mg} / \mathrm{dl}$ were considered hypercholesterolemic. The subjects under anti-hypertensive treatment and those with systolic blood pressure $\geq 140 \mathrm{mmHg}$ and/or diastolic blood pressure $\geq 90 \mathrm{mmHg}$ were considered hypertensive.

Venous blood sampling was performed in the morning, after a 12-h fast. All measurements were performed in the same laboratory and on the same day of sampling, using commercially available kits. In particular, serum levels of NT-proBNP were measured by an electrochemiluminescence immunoassay (proBNP Elecsys, Roche Diagnostics, Mannheim, Germany). The linear range of detection of NT-proBNP was of 5$35,000 \mathrm{pg} / \mathrm{ml}$. The coefficients of variation for intra- and inter-assay measurements of NT-proBNP were, respectively, $4 \%$ and $5 \%$ for "low" mean levels $(210 \mathrm{pg} / \mathrm{ml})$ and $6 \%$ and $7 \%$ for higher mean levels $(4400 \mathrm{pg} / \mathrm{ml})$. The serum levels of high-sensitivity C-reactive protein (CRP) were measured with an immunoturbidimetric method (Tina-quant CRP-Latex, Roche Diagnostics, Mannheim, Germany). The complete blood count was obtained by an automated counter (Bayer ADVIA 120), and erythrocyte sedimentation rate (ESR) was automatically measured by the stopped-flow technique in a capillary microphotometer (Alifax Test 1 System).
Epidemiological and statistical analysis

Participant survival ranged between 22 and 2647 days, with 114 deaths during a median follow-up time of 2458 days (6.7 years), until March 10, 2011. Morbidity was assessed by direct interview of the subject, relatives or caregivers, with assessment also of the available documentation. For disease count, all health problems were recorded either within organ categories (for example, gastroenterological, respiratory, urinary) which were further subdivided into acute and chronic or as previous important diseases (such as myocardial infarction or stroke).

The continuous variables were described as mean \pm standard deviation (SD), or as median and interquartile range, in relation to their distribution (respectively Gaussian or non-Gaussian). The comparisons of such variables between 2 groups were tested, respectively, by Student's $t$ test for unpaired data or Mann-Whitney's $U$ test, while the comparisons among 3 or more groups were assessed with one-way ANOVA and linearity test. The comparisons between percentages were assessed by $\chi^{2}$ test.

Regressions and correlations were assessed by linear regression. For this analysis, the continuous variables with skewed distribution (NT-proBNP, ESR, lymphocytes, leukocytes, creatinine, mean cell volume [MCV], blood glucose and CRP) were preliminarily normalized by natural logarithmic transformation.

The associations with all-cause mortality were assessed by Cox proportional hazards regressions, considering both one variable at a time and all variables together. In these analyses, the Wald statistics was used to compare the strength of association with mortality of the variables. Cox regressions were also used to assess the hazard ratios (HR) and 95\% confidence intervals (CI) associated with variable quintiles, using the low quintile as a reference.

In addition, receiver operating characteristic (ROC) curves with their areas under the curves (AUC) and 95\% CIs were generated to assess the ability of each variable to distinguish deceased subjects from survivors.

Analyses were performed using SPSS Statistics v. 22 (IBM, Armonk, New York, USA). Two-tailed tests were used throughout, and $P$ values $<0.05$ were considered significant.

The main demographic, clinical, and laboratory characteristics of our sample of elderly people are illustrated 
in Table 1, with reference to survivors, deceased, and all subjects.

Table 2 shows the linear regressions and the correlations of chronological age with the laboratory variables. Several variables were preliminarily log-transformed due to their asymmetric log-normal distribution. The variables are listed in decreasing order of Pearson's $r$ coefficient. The logarithm of NT-proBNP was the variable most correlated with age $(r=0.35, P<0.0001)$.

Table 3 shows the associations of the same variables, plus age, and disease count, with all-cause mortality. The associations were assessed by univariate Cox regression, multivariate Cox regression, and area under the ROC curve and are listed in decreasing order of the Wald statistics derived from univariate Cox regression. NT-proBNP was associated with the greatest Wald statistics (both in univariate and multivariate regression) and with the largest AUC. In multivariate Cox regression, NT-proBNP and chronological age were the only variables independently associated with mortality.

According to these assessments, we concluded that the best single indicator of biological age, among laboratory variables, was NT-proBNP. Table 4 confirms that also in our sample, as already known (Redfield et al. 2002; Wang et al. 2002; Luchner et al. 2013), NTproBNP levels were on average higher in women than in men (although the table shows that the difference mainly concerned the "young old" group, namely, the subjects aged 65-74 years). Thus, NT-proBNP values had to be assessed separately in the two sexes.

These are the regression lines of the logarithm of NTproBNP with age, separately for men and women:

$\log (\mathrm{NT}-$ proBNP $)$ men

$$
=0.0827 \times \text { Age }-1.2068(r=0.384, P<0.0001)
$$

$$
\begin{aligned}
& \log (\mathrm{NT}-\text { proBNP }) \text { women } \\
& \quad=0.0478 \times \text { Age }+1.5258(r=0.319, P<0.0001)
\end{aligned}
$$

The intersection point of the two lines can be calculated by solving the equation that is obtained by matching the terms on the right of Eqs. (1) and (2). The result is an age of 78.3 years, corresponding to the NT-proBNP level of $194 \mathrm{pg} / \mathrm{ml}$ for both sexes. Beyond that age, values of men become higher than those of women.
Inverting the two formulas, a possible estimate of biological age can be obtained, in years, starting from an NT-proBNP value. We will call this estimate "proBNP age":

proBNP age men

$$
=[\log (\mathrm{NT}-\text { proBNP })+1.2068] / 0.0827
$$

proBNP age women

$$
=[\log (\mathrm{NT}-\text { proBNP })-1.5258] / 0.0478
$$

The values of proBNP age of the two sexes can now be treated together, and, in fact, Fig. 1 shows that proBNP age distribution is almost perfectly normal, with the same mean of chronological age (72.8 years) but with a larger S.D. (15.9 vs. 5.5 years).

Figure 2 shows the linear relationship between chronological and proBNP age. This is its equation:

$$
\begin{aligned}
\operatorname{proBNP} \text { age }= & 0.999 \times \text { Age } \\
& +0.032(r=0.342, P<0.0001)
\end{aligned}
$$

Since the beta coefficient is very close to 1 and the constant is very close to 0 , the values of proBNP age on the regression line almost coincide with the corresponding values of chronological age, but the variability of the other values is wide.

Figure 3 shows the ROC curve of proBNP age in relation to all-cause mortality, together with the ROC curves of the other laboratory variables most associated with mortality (creatinine, leukocytes, cholesterol, lymphocytes and hemoglobin; see Table 3). The largest area was that under the ROC curve of proBNP age (0.712, 95\% CI 0.661-0.762), confirming, with small increment, the AUC value of NT-proBNP that was calculated without considering sex (see Table 3 ).

Finally, Table 5 shows percent mortality values according to proBNP age quintiles and quintiles of the difference $(\Delta)$ between pro-BNP age and chronological age. Hazard ratios with reference to the lowest quintile are also shown. Considering proBNP age absolute values, mortality increased significantly starting from the third quintile (proBNP age $\geq 69$ years, mortality $7.9 \%$, HR 2.6) and was maximum in the fifth quintile (proBNP age $\geq 85$ years, mortality $22.8 \%$, HR 7.9). Considering the values referred to chronological age 
Table 1 Characteristics of the study sample

\begin{tabular}{|c|c|c|c|c|}
\hline Characteristic & $\begin{array}{l}\text { All } \\
(N=1079)\end{array}$ & $\begin{array}{l}\text { Survivors } \\
(N=965)\end{array}$ & $\begin{array}{l}\text { Deceased } \\
(N=114)\end{array}$ & $P$ value \\
\hline Age (years) & $72.8 \pm 5.5$ & $72.3 \pm 5.0$ & $77.0 \pm 7.0$ & $<0.0001$ \\
\hline Female & $561(52.0)$ & $512(53.1)$ & $49(43.0)$ & 0.04 \\
\hline BMI $\left(\mathrm{kg} / \mathrm{m}^{2}\right)$ & $26.5 \pm 4.0$ & $26.5 \pm 4.1$ & $25.9 \pm 4.0$ & 0.11 \\
\hline Hypertension & $924(85.6)$ & $828(85.8)$ & $96(84.2)$ & 0.65 \\
\hline Hypercholesterolemia & $843(78.1)$ & $765(79.3)$ & $78(68.4)$ & 0.008 \\
\hline Diabetes & $149(13.8)$ & $123(12.7)$ & $26(22.8)$ & 0.003 \\
\hline Ever smoker & $472(43.7)$ & 404 (41.9) & $68(59.6)$ & 0.0003 \\
\hline Previous myocardial infarction & $58(5.4)$ & $48(5.0)$ & $10(8.8)$ & 0.09 \\
\hline Previous stroke & $26(2.4)$ & $19(2.0)$ & $7(6.1)$ & 0.006 \\
\hline Disease count & $1[0-2]$ & $1[0-2]$ & $1[1-2]$ & 0.001 \\
\hline Blood glucose (mg/dl) & $97[90-108]$ & $97[90-108]$ & $97[88-108]$ & 0.56 \\
\hline Cholesterol (mg/dl) & $217.2 \pm 37.6$ & $218.3 \pm 37.3$ & $207.7 \pm 38.6$ & 0.004 \\
\hline Albumin (g/dl) & $4.30 \pm 0.30$ & $4.31 \pm 0.30$ & $4.23 \pm 0.32$ & 0.02 \\
\hline Hemoglobin (g/dl) & $13.9 \pm 1.3$ & $14.0 \pm 1.3$ & $13.6 \pm 1.4$ & 0.001 \\
\hline $\operatorname{MCV}(\mathrm{fl})$ & $89.2[86.3-92.1]$ & 89.2 [86.3-92.0] & 89.1 [85.8-93.3] & 0.71 \\
\hline Leukocytes $\left(\times 10^{9} / 1\right)$ & $5.90[4.96-6.89]$ & $5.86[4.92-6.82]$ & $6.43[5.39-7.46]$ & 0.001 \\
\hline Lymphocytes (\%) & $29.1[24.5-33.7]$ & 29.4 [24.9-33.8] & $27.5[21.4-32.2]$ & 0.003 \\
\hline Creatinine (mg/dl) & $0.94[0.81-1.08]$ & $0.93[0.81-1.07]$ & $1.01[0.87-1.18]$ & 0.0003 \\
\hline ESR (mm/h) & $17[11-30]$ & 17 [11-29] & $23[11-35]$ & 0.01 \\
\hline CRP (mg/dl) & $0.19[0.10-0.39]$ & $0.19[0.09-0.38]$ & $0.20[0.12-0.51]$ & 0.12 \\
\hline NT-proBNP (pg/ml) & $132[75-244]$ & 124 [72-224] & 245 [148-506] & $<0.0001$ \\
\hline
\end{tabular}

Values are mean $\pm \mathrm{SD}$, or median [25th-75th percentile], or number (percentage)

$B M I$ body mass index, $C R P$ C-reactive protein, $E S R$ erythrocyte sedimentation rate, $M C V$ mean cell volume

$(\Delta)$, mortality increased significantly only for values of proBNP age that were 11 or more years greater than chronological age (mortality 20.5\%, HR 3.5). In addition, $\Delta$ proBNP age-chronological age was also associated with morbidity: Fig. 4 shows a progressive rise of this parameter in the presence of 3 or more

Table 2 Linear regressions and correlations with chronological age

\begin{tabular}{lcccc}
\hline Dependent variable & \multicolumn{1}{c}{ Slope } & Intercept & Pearson's r & $P$ value \\
\hline Log (NT-proBNP) & 0.0633 & 0.2983 & 0.350 & $<0.0001$ \\
Hemoglobin & -0.0542 & 17.8879 & 0.230 & $<0.0001$ \\
Log (ESR) & 0.0211 & 1.2860 & 0.149 & $<0.0001$ \\
Log (Lymphocytes) & -0.0063 & 3.8031 & 0.132 & $<0.0001$ \\
Log (Creatinine) & 0.0049 & -0.4120 & 0.123 & $<0.0001$ \\
Albumin & -0.0062 & 4.7332 & 0.081 & 0.008 \\
Cholesterol & -0.5099 & 254.4 & 0.074 & 0.01 \\
Log (MCV) & 0.0009 & 4.4173 & 0.073 & 0.02 \\
Log (Blood Glucose) & -0.0014 & 1.7131 & 0.042 & 0.16 \\
Log (Leukocytes) & -0.0014 & -1.6709 & 0.031 & 0.32 \\
Log (CRP) & 0.0011 & 0.005 & 0.87 \\
\hline
\end{tabular}

$C R P$ C-reactive protein, $E S R$ erythrocyte sedimentation rate, $M C V$ mean cell volume 
Table 3 Relationships with all-cause mortality

\begin{tabular}{|c|c|c|c|c|c|c|}
\hline \multirow[t]{2}{*}{ Independent variable } & \multicolumn{2}{|c|}{ Univariate Cox regression } & \multicolumn{2}{|c|}{ Multivariate Cox regression } & \multicolumn{2}{|c|}{ ROC curve } \\
\hline & Wald* & $P$ value & Wald & $P$ value & AUC & $95 \% \mathrm{CI}$ \\
\hline NT-proBNP & 109.4 & $<0.0001$ & 32.8 & $<0.0001$ & 0.707 & $0.654-0.759$ \\
\hline Chronological age & 70.5 & $<0.0001$ & 30.9 & $<0.0001$ & 0.695 & $0.638-0.752$ \\
\hline Creatinine & 20.7 & $<0.0001$ & 1.5 & 0.23 & 0.603 & $0.545-0.660$ \\
\hline Disease count & 17.5 & 0.0003 & 2.2 & 0.14 & 0.592 & $0.534-0.649$ \\
\hline ESR & 11.6 & 0.0007 & 1.2 & 0.28 & 0.572 & $0.513-0.631$ \\
\hline Hemoglobin & -9.8 & 0.002 & 0.001 & 0.98 & 0.589 & $0.533-0.646$ \\
\hline Lymphocytes & -8.5 & 0.004 & 0.2 & 0.64 & 0.584 & $0.526-0.643$ \\
\hline Cholesterol & -8.4 & 0.004 & 1.1 & 0.29 & 0.582 & $0.524-0.639$ \\
\hline Leukocytes & 7.8 & 0.005 & 3.8 & 0.051 & 0.592 & $0.535-0.649$ \\
\hline CRP & 6.1 & 0.01 & 0.1 & 0.71 & 0.547 & $0.487-0.607$ \\
\hline Albumin & -5.9 & 0.02 & 0.8 & 0.37 & 0.566 & $0.509-0.622$ \\
\hline $\mathrm{MCV}$ & 0.6 & 0.45 & 0.2 & 0.89 & 0.511 & $0.449-0.572$ \\
\hline Blood glucose & 0.2 & 0.65 & 0.1 & 0.81 & 0.483 & $0.422-0.545$ \\
\hline
\end{tabular}

$\overline{A U C}$ area under curve, $C R P$ C-reactive protein, $E S R$ erythrocyte sedimentation rate, $M C V$ mean cell volume, $R O C$ receiver operating characteristic

*Minus signs denote inverse relationships. The AUCs of the variables inversely associated with mortality have been symmetrically inverted to allow direct comparison with other AUCs

diseases $(P$ ANOVA $<0.0001, P$ for linear trend $=$ $0.0002)$.

\section{Discussion}

In this study, among several biochemical health and disease indicators, NT-proBNP was the one most correlated with chronological age and allcause mortality (in the case of mortality, with even better results than chronological age and disease count). Thus, using simple formulas separately for the two sexes, starting from NT-proBNP values, we obtained an estimate of biological age, proBNP age, which, unlike NT-proBNP, can be considered equally valid for men and women.
ProBNP age is probably not an accurate measure of real biological age, but it may equally serve as surrogate end point in epidemiological studies and even for personal use: (1) it is easily interpretable, (2) it may be assessed longitudinally to study its course (spontaneous, or possibly influenced by treatment), and (3) it is a measure of the risk of morbidity and all-cause death. In particular, anyone can calculate his/her proBNP age, according to his/her NT-proBNP value and sex, using the coefficients provided in the Results (formulas (3) and (4)). Of course, having a proBNP age equal or younger than chronological age will be preferable, although significantly increased mortality was only associated with an excess of 11 or more years with respect to chronological age. In absolute, the lowest risk of death was associated with a proBNP age $<69$ years, while the

Table 4 NT-pro BNP values according to age and sex

\begin{tabular}{lllr}
\hline Age group & Men & Women & $P$ value \\
\hline All ages & $119[59-238](N=518)$ & $143[91-246](N=561)$ & 0.0001 \\
$65-74$ years & $98[52-179](N=379)$ & $128[82-214](N=383)$ & $<0.0001$ \\
$75-84$ years & $201[99-426](N=127)$ & $198[110-359](N=157)$ & 0.89 \\
$\geq 85$ years & $361[241-583](N=12)$ & $277[197-393](N=21)$ & 0.41 \\
\hline
\end{tabular}

Values, in picograms per milliliter, are medians [25th-75th percentile] 
Fig. 1 Histogram of proBNP age, showing an almost normal distribution
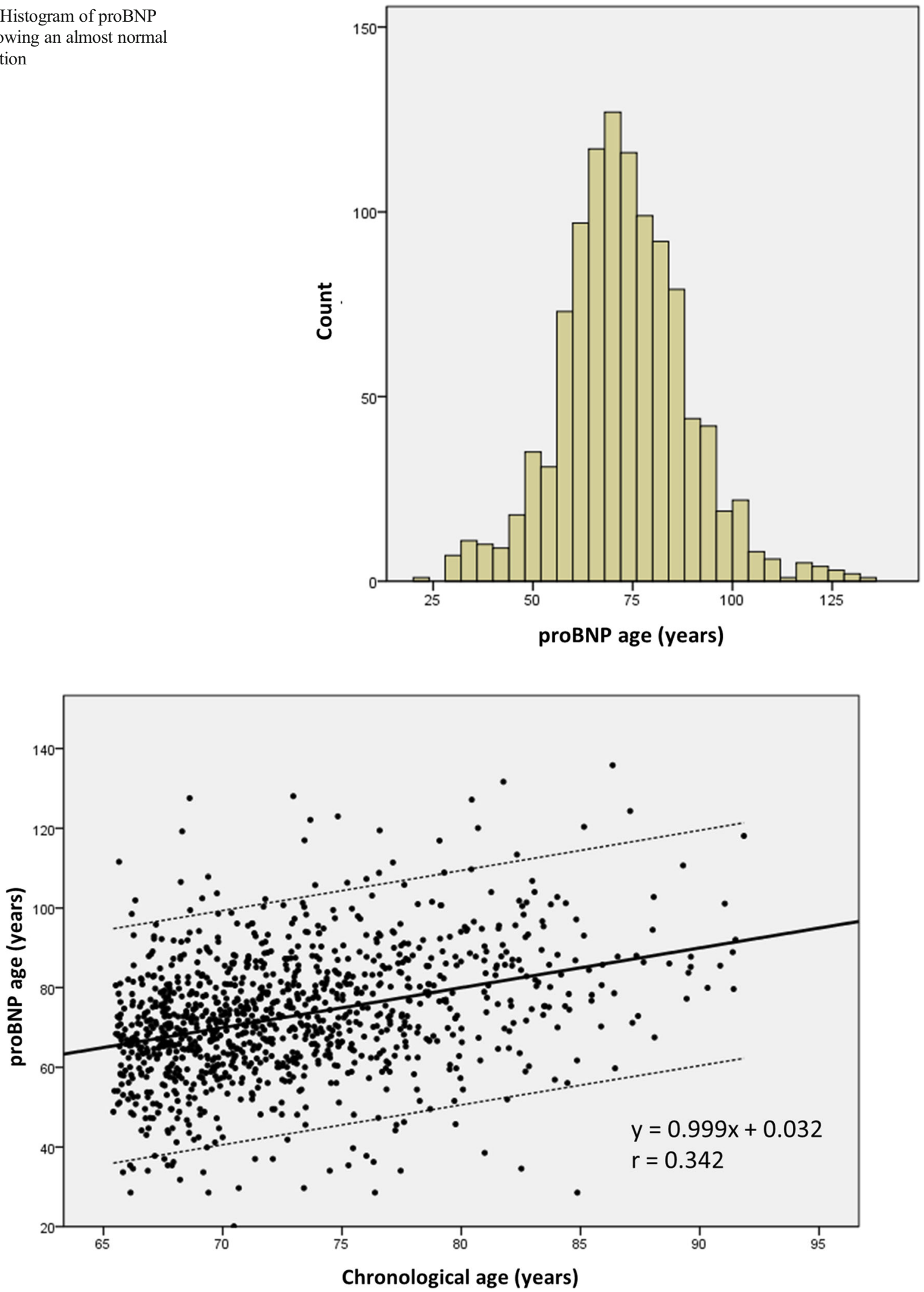

Fig. 2 Regression line and 95\% confidence interval between chronological age and proBNP age 
Fig. 3 Receiver operating characteristic (ROC) curves of proBNP age (area under the curve (AUC) $0.712,95 \%$ CI $0.661-$ 0.762 ) and of the variables most associated with all-cause mortality: creatinine (AUC 0.603, 95\% CI $0.546-0.660)$, leukocytes (AUC 0.592, 95\% CI 0.5350.649), cholesterol (AUC 0.419, 95\% CI 0.361-0.476), lymphocytes (AUC $0.416,95 \%$ CI 0.357-0.474), and hemoglobin (AUC 0.411, 95\% CI 0.354$0.468)$

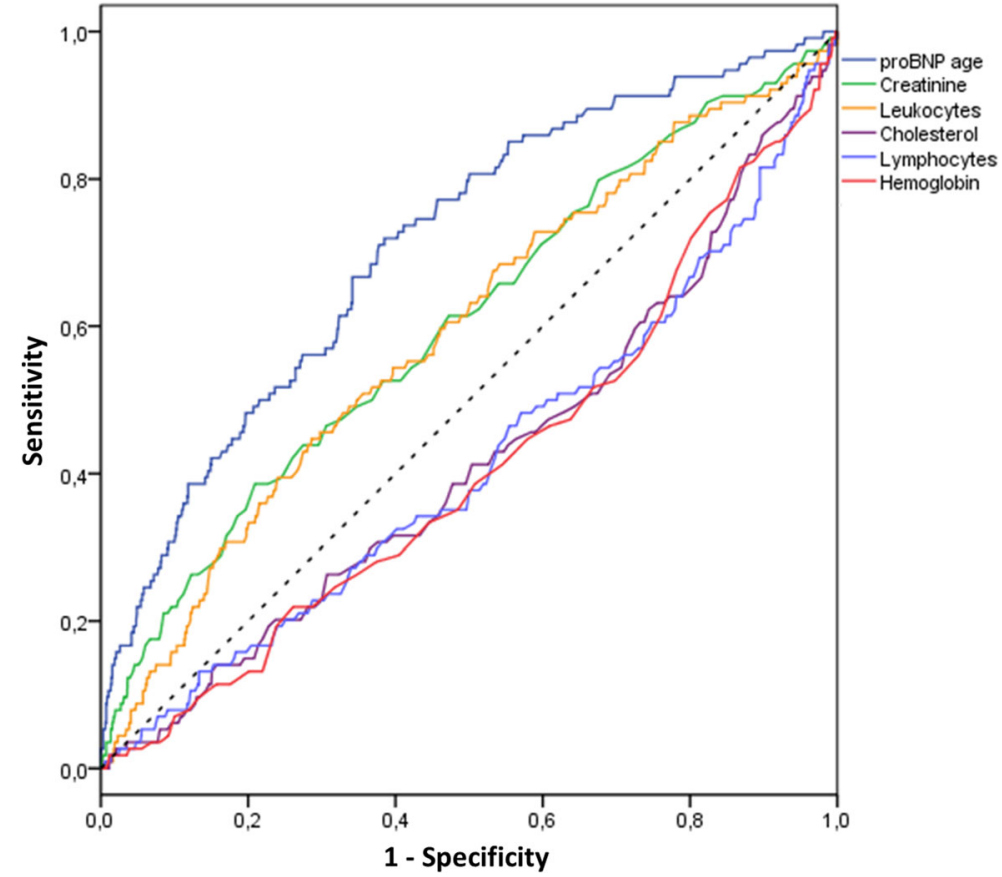

highest risk was associated with a proBNP age $\geq$ 85 years.

Non-cardiovascular determinants of NT-proBNP and association with non-cardiovascular mortality

NT-proBNP, like BNP, is released from cardiomyocytes undergoing wall stress or ischemia and, therefore, it is a known indicator of heart failure, atrial fibrillation, acute coronary syndromes, and cardiovascular mortality (McDonagh et al. 2004; Galvani et al. 2004; Ponikowski et al. 2016; Wang et al. 2004; Ulimoen et al. 2009).
Other cardiovascular factors directly associated with an increase in these natriuretic peptides are systemic and pulmonary arterial hypertension, myocardial hypertrophy, pulmonary embolism, myocarditis, valve and congenital heart diseases, tachyarrhythmias, electric cardioversion, and betablocker treatment (Ponikowski et al. 2016), while heart rate is inversely correlated (Loke et al. 2003). However, several studies have shown that these natriuretic peptides are also associated with non-cardiovascular mortality (Muscari et al. 2013) and all-cause mortality in subjects free of cardiovascular diseases (McKie et al. 2006;

Table 5 All-cause mortality according to proBNP age quintiles (absolute values or values referred to chronological age)

\begin{tabular}{lllllllrrr} 
proBNP age quintile & Number & Deceased Percent & HR $(95 \%$ CI $)$ & $\begin{array}{l}\Delta \text { proBNP } \\
\text { age-chronological } \\
\text { age quintile }\end{array}$ & Number Deceased Percent HR $(95 \%$ CI $)$ \\
\hline $5(\geq 85$ years $)$ & 224 & 51 & 22.8 & $7.9(3.6-17.5)$ & $5(\geq 11$ years $)$ & 220 & 45 & 20.5 & $3.5(1.9-6.4)$ \\
$4(\geq 75,<85$ years $)$ & 228 & 31 & 13.6 & $4.5(2.0-10.2)$ & $4(\geq 3,<11$ years $)$ & 224 & 17 & 7.6 & $1.2(0.6-2.4)$ \\
$3(\geq 69,<75$ years $)$ & 189 & 15 & 7.9 & $2.6(1.0-6.3)$ & $3(\geq-3,<3$ years $)$ & 198 & 22 & 11.1 & $1.8(0.9-3.5)$ \\
$2(\geq 61,<69$ years $)$ & 219 & 10 & 4.6 & $1.4(0.5-3.8)$ & $2(\geq-11,<-3$ years $)$ & 216 & 16 & 7.4 & $1.2(0.6-2.4)$ \\
$1(<61$ years $)$ & 219 & 7 & 3.2 & 1 & $1(<-11$ years $)$ & 221 & 14 & 6.3 & 1
\end{tabular}

Hazard ratios are referred to first quintile

$C I$ confidence interval, $H R$ hazard ratio 


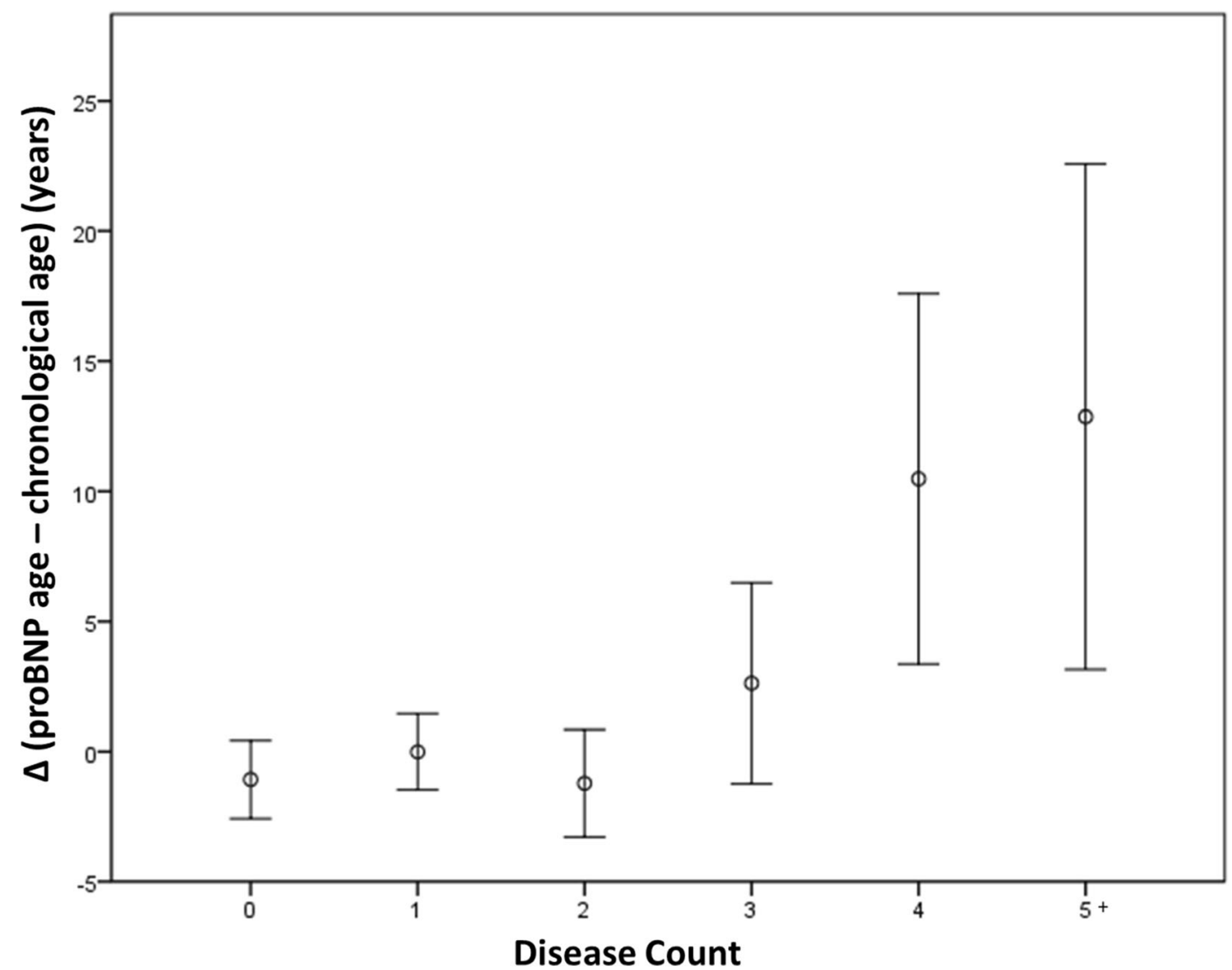

Fig. 4 Relationship between $\Delta$ proBNP age-chronological age (mean values \pm S.E.M.) and disease count

Wannamethee et al. 2011; Muscari et al. 2013), and the same occurred in this study, from which the subjects with a history of heart failure and atrial fibrillation had been excluded. In fact, also many extra-cardiac determinants are associated with an increment of these natriuretic peptides, such as renal insufficiency, anemia, diabetes, ischemic stroke, subarachnoid hemorrhage, paraneoplastic syndromes, chronic obstructive pulmonary disease (COPD), sepsis, burns, severe hormonal dysfunctions, and liver diseases like cirrhosis (Ponikowski et al. 2016), while obesity, especially visceral, and hepatic steatosis are associated with lower serum levels (Luchner et al. 2013; Muscari et al. 2006). The association between NT-proBNP levels and non-cardiovascular mortality might also have simpler explanations, in addition to the above non-cardiac determinants, such as the concomitance of inapparent myocardial impairment, or coding errors in death certificates.
Association between age and NT-proBNP

The levels of NT-proBNP progressively increase with age even in healthy subjects (Redfield et al. 2002; Wang et al. 2002), as was also confirmed by a long-term longitudinal study (Luchner et al. 2013). The relationship between age and NT-proBNP is of exponential type (Redfield et al. 2002; Luchner et al. 2013) (it becomes linear after logarithmic transformation of values), like the relationship between age and mortality (at least before the age of 105 years) (Barbi et al. 2018). The European guidelines for heart failure suggest a unique cutoff of NT-proBNP, independent of age, below which the probability of heart failure is considered neglectable: $125 \mathrm{pg} / \mathrm{ml}$ for non-acute heart failure and $300 \mathrm{pg} / \mathrm{ml}$ for acute heart failure (Ponikowski et al. 2016). Nevertheless, in our sample, the first cutoff was exceeded, on average, at the age of 69 by women and of 73 by men, and the second cutoff was exceeded at the age of 87 by women and of 83 by men, in the absence of heart failure. 
Indeed, several authors have suggested to adopt different cutoffs for the diagnosis of heart failure, taking into account the progressive increase of natriuretic peptides with age (Nageh et al. 2002; Hildebrandt et al. 2010; Keyzer et al. 2014). The reason for this age-dependent increment in both sexes is unknown. It might be related to aging-associated processes, such as myocardial hypertrophy and fibrosis (Boyle et al. 2011), or the reduction of clearing mechanisms of natriuretic peptides from plasma (Clark et al. 1991).

\section{Association between female sex and NT-proBNP}

The association of higher levels of NT-proBNP with female sex is well known (Redfield et al. 2002; Loke et al. 2003), so that some laboratories have proposed differentiated normal values for the two sexes. In our sample, the regression line between age and logarithm of NT-proBNP in women had an intercept greater than that of men, so that, for the major part of life, for the same age, women had higher NT-proBNP levels and, for the same NT-proBNP level, they were younger than men. However, the regression line in men was steeper than that in women, so after the age of 78.3, the values in men exceeded those in women. To our knowledge, this is the first time that the overtaking of NT-proBNP values in women by values in men is documented, although Raymond et al. in 2003 reported a significant difference, in favor of women, only up to 70 years of age (Raymond et al. 2003) and, more recently, in a sample with a mean age lower than that of our sample, higher values in women were documented up to 50 years of age (Hamada et al. 2017). In general, the causes of the different NT-proBNP levels in the two sexes are poorly understood. In a previous study, we found that NTproBNP levels are inversely related to hematocrit, which is lower in women than in men (Muscari et al. 2006). Other possible explanations concern the lower levels of plasma renin in women (Kuroski de Bold 1999) or the effects that female sex hormones may have on gene expression of natriuretic peptides (Hong et al. 1992).

Is it possible to modify blood levels of natriuretic peptides with anti-aging interventions?

In patients with heart failure and high natriuretic peptide levels, it is certainly possible to obtain a decrement of such levels with heart failure treatment (Richards and Troughton 2004). However, in subjects without heart disease, it is unknown whether reducing these peptide levels, or at least slowing their progression, is possible with anti-aging treatments, also, and mainly because at present such treatments are only hypothetical. The only anti-aging treatment of documented efficacy is caloric restriction with adequate nutrition (Dorling et al. 2020). In rat, regimens of dietetic restriction prevented the agerelated increase of ANP stores in atrial tissue and lowered plasma ANP levels (Cavallini et al. 1995). To our knowledge, there are no equivalent studies in man concerning BNP or NT-proBNP.

\section{Other findings}

As far as the other laboratory parameters considered are concerned, creatinine, after NT-proBNP, was the variable most associated with mortality, in addition of being significantly correlated with age, as already reported by others (Liu et al. 2018). In addition, the inverse association of hemoglobin, lymphocytes, cholesterol, and albumin with both age and mortality was also confirmed, probably because in elderly people, low values of these variables are markers of frailty. However, in a multivariate analysis including NT-proBNP, none of these laboratory variables was independently associated with mortality.

Previous studies on laboratory or clinical variables and biological age

The attempts to obtain an indicator of biological age starting from laboratory or clinical, usually multiple, variables, have been numerous. Sometimes indices exclusively composed by clinical-anamnestic data, without any laboratory variable, have been proposed, such as the deficit index (FI34) by Kim and Jazwinski (2015). This index was based on 34 variables, such as anemia history, cataract history, asthma history, COPD history, kidney disease history, BMI, Mini Mental State Examination, Activities of Daily Living, Geriatric Depression Scale, and self-assessment of health status. This index was well associated with mortality and disease count, which is understandable considering that it includes clinical-anamnestic references to all main human pathologies. It is certainly an accurate assessment of health, frailty, and comorbidity status, which however is more associated with the effects of diseases rather than with the aging process. In addition, considering the prevalence of anamnestic parameters (24 out of 34 ), it 
might be poorly influenced by possible treatments. A similar and even more complex index (40 items) of multidimensional frailty has been proposed for the Italian elderly population (Abete et al. 2017). This index also includes Instrumental Activities of Daily Living, weight loss, peak expiratory flow, Mini Nutritional Assessment, and an accurate evaluation of walking ability and muscle strength, with good associations with mortality, disability, and hospitalization rate.

On the other hand, other studies utilized common laboratory parameters, such as creatinine, CRP, albumin, cholesterol, and leukocytes, which were combined by mathematical functions to produce biological age directly or indirectly through intermediate calculation of mortality (Yoo et al. 2017; Belsky et al. 2017; Liu et al. 2018; Waziry et al. 2019). Sometimes, systolic blood pressure was included in the index (Belsky et al. 2017; Waziry et al. 2019), although, considering the spontaneous variability of this parameter, random variations of biological age could be generated. In addition, systolic blood pressure increases with age, but its relationship with mortality, in the elderly and in some pathologies, may be of inverse type (Muscari et al. 2013; Weiss et al. 2010; Okin et al. 2012). In some cases, among the predictive parameters of biological age, also chronological age was included, thus obtaining very good correlations between chronological age and the index of biological age, which might be, however, a biased procedure (Liu et al. 2018). These indices have at least the potentiality to improve with some form of anti-aging treatment, and, in fact, after 2 years of caloric restriction, in the CALERIE trial, Belsky et al. (2017) obtained an improvement of two indices of biological age that were mainly based on biochemical variables. However, cholesterol, uric acid, blood glucose, and systolic blood pressure may also be influenced by drugs, which may cause relevant fluctuations in the calculation of biological age according to Belsky's formulas. In addition, very reliable and validated multiparametric risk profiles have been available for many years (D’Agostino et al. 2008), and the transformation of a multiparametric mortality risk into years of biological age might appear a useless procedure. Finally, none of these attempts to estimate biological age included BNP or NT-proBNP, which carry a great part of the information associated with other biochemical parameters. In fact, we have documented that the association with mortality of many of these parameters disappeared when the multivariate analysis included NT-proBNP, thus making useless the creation of a complicate multiparametric index.

\section{Limitations}

First of all, this study needs a confirmation, being the first study that has proposed NT-proBNP as a possible indicator of biological age. Thus, it would be advisable to seek to reproduce our assessments in a different and possibly larger population. In addition, the associations of pro-BNP age with diseaseassociated mortalities and specific pathologies should be verified. It would also be desirable a direct comparison with other modalities of biological age assessment. Probably, proBNP age cannot reliably be calculated in subjects with very high NTproBNP levels, as is the case in the presence of heart failure or atrial fibrillation. There is also the need to extend the assessment to younger subjects (the relationships found in people over 65 years of age cannot automatically be extrapolated downwards). Finally, we found that NT-proBNP is probably the single best predictor of mortality and that it is also associated with disease count, but whether it truly reflects some of the biological mechanisms of aging is uncertain and its relationship with other agerelated outcomes, such as reduced walking speed, physical disability, and cognitive impairment, should be assessed in further studies.

\section{Conclusions}

Among all the laboratory variables considered in this study, NT-proBNP was the one most associated with chronological age and all-cause mortality. Thus, through simple formulas, we have proposed a method that allows the transformation of NTproBNP, which varies differently in the two sexes, into a value of "pro-BNP age," which is independent of sex and easily interpretable both as absolute value and in relation to chronological age. If confirmed by other studies, this indicator of biological age, obtained by a simple blood measurement, might prove useful as a surrogate end point in the assessment of anti-aging treatments. 
Funding information Open access funding provided by Alma Mater Studiorum - Università di Bologna within the CRUI-CARE Agreement. The study was supported by grants from Fondazione Cassa di Risparmio in Bologna and Regione Emilia RomagnaPiani per la Salute.

Data availability The data that support the findings of this study are available from the corresponding author upon reasonable request.

\section{Compliance with ethical standards}

Conflict of interest The authors declare that they have no conflict of interest.

Ethics approval The study was approved by the joint Ethical Committee of the University of Bologna and S.Orsola Hospital, Bologna, Italy.

Consent to participate All participants provided their signed informed consent.

Open Access This article is licensed under a Creative Commons Attribution 4.0 International License, which permits use, sharing, adaptation, distribution and reproduction in any medium or format, as long as you give appropriate credit to the original author(s) and the source, provide a link to the Creative Commons licence, and indicate if changes were made. The images or other third party material in this article are included in the article's Creative Commons licence, unless indicated otherwise in a credit line to the material. If material is not included in the article's Creative Commons licence and your intended use is not permitted by statutory regulation or exceeds the permitted use, you will need to obtain permission directly from the copyright holder. To view a copy of this licence, visit http://creativecommons.org/licenses/by/4.0/.

\section{References}

Abete P, Basile C, Bulli G, Curcio F, Liguori I, Della-Morte D, et al. The Italian version of the "frailty index" based on deficits in health: a validation study. Aging Clin Exp Res. 2017;29:913-26.

Barbi E, Lagona F, Marsili M, Vaupel JW, Wachter KW. The plateau of human mortality: demography of longevity pioneers. Science. 2018;360:1459-61.

Belsky DW, Huffman KM, Pieper CF, Shalev I, Kraus WE. Change in the rate of biological aging in response to caloric restriction: CALERIE Biobank Analysis. J Gerontol A Biol Sci Med Sci. 2017;73:4-10.

Boyle AJ, Shih H, Hwang J, Ye J, Lee B, Zhang Y, et al. Cardiomyopathy of aging in the mammalian heart is characterized by myocardial hypertrophy, fibrosis and a predisposition towards cardiomyocyte apoptosis and autophagy. Exp Gerontol. 2011;46:549-59.
Cavallini G, Clerico A, Del Chicca M, Gori Z, Bergamini E. Effects of different types of anti-aging dietary restrictions on age-related atrial natriuretic factor changes: an immunochemical and ultrastructural study. Aging (Milano). 1995;7: $117-22$.

Chen BH, Marioni RE, Colicino E, Peters MJ, Ward-Caviness CK, Tsai PC, et al. DNA methylation-based measures of biological age: meta-analysis predicting time to death. Aging (Albany NY). 2016;8:1844-65.

Clark BA, Elahi D, Shannon RP, Wei JY, Epstein FH. Influence of age and dose on the end-organ responses to atrial natriuretic peptide in humans. Am J Hypertens. 1991;4:500-7.

Cole JH, Ritchie SJ, Bastin ME, Valdés Hernández MC, Muñoz Maniega S, Royle N, et al. Brain age predicts mortality. Mol Psychiatry. 2018;23:1385-92.

D’Agostino RB Sr, Vasan RS, Pencina MJ, Wolf PA, Cobain M, Massaro JM, et al. General cardiovascular risk profile for use in primary care: the Framingham Heart Study. Circulation. 2008;117:743-53.

Dorling JL, Martin CK, Redman LM. Calorie restriction for enhanced longevity: the role of novel dietary strategies in the present obesogenic environment. Ageing Res Rev. 2020: 101038. https://doi.org/10.1016/j.arr.2020.101038.

Elliott ML, Belsky DW, Knodt AR, Ireland D, Melzer TR, Poulton R, et al. Brain-age in midlife is associated with accelerated biological aging and cognitive decline in a longitudinal birth cohort. Mol Psychiatry. 2019. https://doi. org/10.1038/s41380-019-0626-7.

Galvani M, Ferrini D, Ottani F. Natriuretic peptides for risk stratification of patients with acute coronary syndromes. Eur J Heart Fail. 2004;6:327-33.

Hamada M, Shigematsu Y, Takezaki M, Ikeda S, Ogimoto A. Plasma levels of atrial and brain natriuretic peptides in apparently healthy subjects: effects of sex, age, and hemoglobin concentration. Int J Cardiol. 2017;228:599-604.

Hannum G, Guinney J, Zhao L, Zhang L, Hughes G, Sadda S, et al. Genome-wide methylation profiles reveal quantitative views of human aging rates. Mol Cell. 2013;49:359-67.

Hildebrandt P, Collinson PO, Doughty RN, Fuat A, Gaze DC, Gustafsson F, et al. Age-dependent values of N-terminal proB-type natriuretic peptide are superior to a single cut-point for ruling out suspected systolic dysfunction in primary care. Eur Heart J. 2010;31:1881-9.

Hong M, Yan Q, Tao B, Boersma A, Han KK, Vantyghem MC, et al. Estradiol, progesterone and testosterone exposures affect the atrial natriuretic peptide gene expression in vivo in rats. Biol Chem Hoppe Seyler. 1992;373:213-8.

Horvath S. DNA methylation age of human tissues and cell types. Genome Biol. 2013;14:R115.

Johnson TE. Recent results: biomarkers of aging. Exp Gerontol. 2006;41:1243-6.

Johnson LC, Parker K, Aguirre BF, Nemkov TG, D'Alessandro A, Johnson SA, et al. The plasma metabolome as a predictor of biological aging in humans. Geroscience. 2019;41:895906.

Jylhävä J, Pedersen NL, Hägg S. Biological age predictors. EBioMedicine. 2017;21:29-36.

Keyzer JM, Hoffmann JJ, Ringoir L, Nabbe KC, Widdershoven JW, Pop VJ. Age- and gender-specific brain natriuretic peptide (BNP) reference ranges in primary care. Clin Chem Lab Med. 2014;52:1341-6. 
Kim S, Jazwinski SM. Quantitative measures of healthy aging and biological age. Healthy Aging Res. 2015;4:26.

Kuroski de Bold ML. Estrogen, natriuretic peptides and the reninangiotensin system. Cardiovasc Res. 1999;41:524-31.

Liu Z, Kuo PL, Horvath S, Crimmins E, Ferrucci L, Levine M. A new aging measure captures morbidity and mortality risk across diverse subpopulations from NHANES IV: a cohort study. PLoS Med. 2018;15:e1002718. https://doi. org/10.1371/journal.pmed.1002718.

Loke I, Squire IB, Davies JE, Ng LL. Reference ranges for natriuretic peptides for diagnostic use are dependent on age, gender and heart rate. Eur J Heart Fail. 2003;5:599-606.

Luchner A, Behrens G, Stritzke J, Markus M, Stark K, Peters A, et al. Long-term pattern of brain natriuretic peptide and $\mathrm{N}$ terminal pro brain natriuretic peptide and its determinants in the general population: contribution of age, gender, and cardiac and extra-cardiac factors. Eur J Heart Fail. 2013;15: 859-67.

McDonagh TA, Holmer S, Raymond I, Luchner A, Hildebrant P, Dargie HJ. NT-proBNP and the diagnosis of heart failure: a pooled analysis of three European epidemiological studies. Eur J Heart Fail. 2004;6:269-73.

McKie PM, Rodeheffer RJ, Cataliotti A, Martin FL, Urban LH, Mahoney DW, et al. Amino-terminal pro-B-type natriuretic peptide and B-type natriuretic peptide: biomarkers for mortality in a large community-based cohort free of heart failure. Hypertension. 2006;47:874-80.

Menni C, Kiddle SJ, Mangino M, Viñuela A, Psatha M, Steves C, et al. Circulating proteomic signatures of chronological age. $\mathrm{J}$ Gerontol A Biol Sci Med Sci. 2015;70:809-16.

Mitnitski AB, Mogilner AJ, Rockwood K. Accumulation of deficits as a proxy measure of aging. Sci World J. 2001;1:32336.

Muscari A, Berzigotti A, Bianchi G, Giannoni C, Ligabue A, Magalotti D, et al. Non-cardiac determinants of NTproBNP levels in the elderly: relevance of haematocrit and hepatic steatosis. Eur J Heart Fail. 2006;8:468-76.

Muscari A, Antonelli S, Bianchi G, Cavrini G, Dapporto S, Ligabue A, et al. Serum C3 is a stronger inflammatory marker of insulin resistance than C-reactive protein, leukocyte count, and erythrocyte sedimentation rate: comparison study in an elderly population. Diabetes Care. 2007;30: 2362-8.

Muscari A, Bianchi G, Forti P, Giansante C, Giovagnoli M, Magalotti D, et al. A comparison of risk factors as predictors of cardiovascular and non-cardiovascular mortality in the elderly people-relevance of N-terminal pro-B-type natriuretic peptide and low systolic blood pressure. Int J Clin Pract. 2013;67:1182-91.

Nageh T, Chin D, Cooke JC, Meehan N, Monaghan MJ, Sherwood RA. Interpretation of plasma brain natriuretic peptide concentrations may require adjustment for patient's age. Ann Clin Biochem. 2002;39:151-3.

Okin PM, Hille DA, Kjeldsen SE, Dahlöf B, Devereux RB. Impact of lower achieved blood pressure on outcomes in hypertensive patients. J Hypertens. 2012;30:802-10.

Perna L, Zhang Y, Mons U, Holleczek B, Saum KU, Brenner H. Epigenetic age acceleration predicts cancer, cardiovascular, and all-cause mortality in a German case cohort. Clin
Epigenetics. 2016;8:64. https://doi.org/10.1186/s13148016-0228-z.

Ponikowski P, Voors AA, Anker SD, Bueno H, Cleland JG, Coats AJ, et al. 2016 ESC guidelines for the diagnosis and treatment of acute and chronic heart failure: the Task Force for the diagnosis and treatment of acute and chronic heart failure of the European Society of Cardiology (ESC). Developed with the special contribution of the Heart Failure Association (HFA) of the ESC. Eur J Heart Fail. 2016;18:891-975.

Raymond I, Groenning BA, Hildebrandt PR, Nilsson JC, Baumann M, Trawinski J, et al. The influence of age, sex and other variables on the plasma level of $\mathrm{N}$-terminal pro brain natriuretic peptide in a large sample of the general population. Heart. 2003;89:745-51.

Redfield MM, Rodeheffer RJ, Jacobsen SJ, Mahoney DW, Bailey KR, Burnett JC Jr. Plasma brain natriuretic peptide concentration: impact of age and gender. J Am Coll Cardiol. 2002;40:976-82.

Richards M, Troughton RW. NT-proBNP in heart failure: therapy decisions and monitoring. Eur J Heart Fail. 2004;6:351-4.

Rockwood K, Blodgett JM, Theou O, Sun MH, Feridooni HA, Mitnitski A, et al. A frailty index based on deficit accumulation quantifies mortality risk in humans and in mice. Sci Rep. 2017;7:43068. https://doi.org/10.1038/srep43068.

Sanders JL, Newman AB. Telomere length in epidemiology: a biomarker of aging, age-related disease, both, or neither? Epidemiol Rev. 2013;35:112-31.

Ulimoen SR, Enger S, Tveit A. Impact of atrial fibrillation on NTproBNP levels in a 75-year-old population. Scand J Clin Lab Invest. 2009;69:579-84.

Wang TJ, Larson MG, Levy D, Leip EP, Benjamin EJ, Wilson PW, et al. Impact of age and sex on plasma natriuretic peptide levels in healthy adults. Am J Cardiol. 2002;90:254-8.

Wang TJ, Larson MG, Levy D, Benjamin EJ, Leip EP, Omland T, et al. Plasma natriuretic peptide levels and the risk of cardiovascular events and death. N Engl J Med. 2004;350:655-63.

Wannamethee SG, Welsh P, Lowe GD, Gudnason V, Di Angelantonio E, Lennon L, et al. N-terminal pro-brain natriuretic peptide is a more useful predictor of cardiovascular disease risk than C-reactive protein in older men with and without pre-existing cardiovascular disease. J Am Coll Cardiol. 2011;58:56-64.

Waziry R, Gras L, Sedaghat S, Tiemeier H, Weverling GJ, Ghanbari M, et al. Quantification of biological age as a determinant of age-related diseases in the Rotterdam Study: a structural equation modeling approach. Eur J Epidemiol. 2019;34:793-9.

Weiss JW, Johnson ES, Petrik A, Smith DH, Yang X, Thorp ML. Systolic blood pressure and mortality among older community-dwelling adults with CKD. Am J Kidney Dis. 2010;56:1062-71.

Yoo J, Kim Y, Cho ER, Jee SH. Biological age as a useful index to predict seventeen-year survival and mortality in Koreans. BMC Geriatr. 2017;17:7. https://doi.org/10.1186/s12877016-0407-y.

Publisher's note Springer Nature remains neutral with regard to jurisdictional claims in published maps and institutional affiliations. 